\title{
Quantum Melting of Magnetic Order due to Orbital Fluctuations
}

\author{
Louis Felix Feiner \\ Philips Research Laboratories, Prof. Holstlaan 4, NL-5656 AA Eindhoven, The Netherlands \\ Andrzej M. Oleś* \\ Max-Planck-Institut für Festkörperforschung, Heisenbergstrasse 1, D-70569 Stuttgart, Federal Republic of Germany \\ Jan Zaanen \\ Lorentz Institute for Theoretical Physics, Leiden University, P.O.B. 9506, NL-2300 RA Leiden, The Netherlands
}

(Received 27 November 1996)

\begin{abstract}
We have studied the phase diagram and excitations of the spin-orbital model derived for a three-dimensional perovskite lattice, as in $\mathrm{KCuF}_{3}$. The results demonstrate that the orbital degeneracy drastically increases quantum fluctuations and suppresses the classical long-range order near the multicritical point in the mean-field phase diagram. This indicates the presence of a quantum liquid state, and we present explicit evidence for valence bond type correlations in three dimensions. [S0031-9007(97)02824-X]
\end{abstract}

PACS numbers: 71.27.+a, 75.10.-b, 74.72.-h, 75.30.Et

It is common knowledge that macroscopic ensembles of interacting particles tend to behave classically. This is not always true, however, and the study of collective quantum systems starts to become a prominent theme in condensed matter physics. Central to this pursuit are low-dimensional quantum spin systems (spin chains and ladders [1]), and it proves difficult to achieve quantum melting of magnetic long-range order (LRO) in empirically relevant systems in higher dimensions. Here we suggest a class of systems in which quantum melting occurs even in three dimensions: small spin, orbital degenerate magnetic insulators, and the so-called KugelKhomskii (KK) systems [2]. There might already exist a physical realization of such a three-dimensional (3D) "quantum spin-orbital liquid": $\mathrm{LiNiO}_{2}$.

Global SU(2) by itself is not symmetric enough to defeat classical order in $D>1$, and the pursuit has been open for some time to engineer more fluctuations into these systems. Three (related) strategies to realize quantum melting have proven to be successful: (i) adding zero-dimensional fluctuations as in the bilayer Heisenberg model which leads to an incompressible spin liquid $[3,4]$, (ii) frustrating the system so that the classical sector gets highly degenerate, as in the case of the $S=1 / 2$ square lattice with longer ranged antiferromagnetic (AF) interactions $\left(J_{1}-J_{2}-J_{3}\right.$ models $\left.[5,6]\right)$. These systems involve fine-tuning of parameters and are therefore hard to realize by chemistry. (iii) Finally, the third strategy would be to reduce the number of magnetic bonds, as in the 1/5-depleted square lattice, where the resulting plaquette resonating valence bond (PRVB) state explains the spin gap observed in $\mathrm{CaV}_{4} \mathrm{O}_{9}$ [7]. In this Letter we show that orbital degeneracy operates through the same basic mechanisms to produce quantum melting in the KK systems. The novelty is that these systems tend to "self-tune" to (critical) points of high classical degeneracy. There are interactions which may lift the classical degeneracy, but they are usually weak.

An interaction of this kind is the electron-phonon coupling - the degeneracy is lifted by a change in crystal structure, the conventional collective Jahn-Teller (JT) instability. However, as was pointed out in the seminal work by Kugel and Khomskii [2], in orbital degenerate Mott-Hubbard insulators one has to consider in the first instance the purely electronic problem. Because of the large local Coulomb interactions (Hubbard $U$ ), a low energy Hilbert space splits off, spanned by spin and orbital configuration space, with superexchangelike couplings between both spin and orbital local degrees of freedom. The orbital sector carries a discrete symmetry, and the net outcome is that the clocklike orbital degrees of freedom get coupled into the SU(2) spin problem. Such a system might undergo symmetry breaking in states with simultaneous spin and orbital order. The lattice has to react to the symmetry lowering in the orbital sector, but it was recently shown, at least in the archetypical compound $\mathrm{KCuF}_{3}$, that such a distortion is a side effect [8].

The fundamental question arises if these forms of classical order are always stable against quantum fluctuations. Although the subject is much more general (singlet-triplet models in rare earth compounds [9], $\mathrm{V}_{2} \mathrm{O}_{3}$ [10], $\mathrm{LaMnO}_{3}$ [11], heavy fermions [12]), we focus here on the simplest situation encountered in $\mathrm{KCuF}_{3}$ and related systems [2]. These are JT-distorted cubic, 3D analogs of the cuprate superconductors [13]. The magnetic ion is in a $3 d^{9}$ state, characterized in the absence of JT distortion by two degenerate $e_{g}\left(x^{2}-y^{2} \sim|x\rangle, 3 z^{2}-r^{2} \sim|z\rangle\right)$ orbitals next to the $S=1 / 2$ spin degeneracy. Kugel and Khomskii derived the effective Hamiltonian [2] with AF superexchange $J=t^{2} / U$ (where $t$ is the hopping between 
$|z\rangle$ orbitals along the $c$ axis),

$$
\begin{array}{r}
H_{1}=J \sum_{\langle i j\rangle}\left[4\left(\vec{S}_{i} \cdot \vec{S}_{j}\right)\left(\tau_{i}^{\alpha}-\frac{1}{2}\right)\left(\tau_{j}^{\alpha}-\frac{1}{2}\right)\right. \\
\left.+\left(\tau_{i}^{\alpha}+\frac{1}{2}\right)\left(\tau_{j}^{\alpha}+\frac{1}{2}\right)-1\right],
\end{array}
$$

neglecting the Hund's rule splittings of the intermediate $d^{8}$ states. Including those up to order $\eta=J_{H} / U\left(J_{H}\right.$ is the singlet-triplet splitting) yields, in addition,

$$
\begin{aligned}
H_{2}=J \eta \sum_{\langle i j\rangle}[ & \left(\vec{S}_{i} \cdot \vec{S}_{j}\right)\left(\tau_{i}^{\alpha}+\tau_{j}^{\alpha}-1\right) \\
& +\frac{1}{2}\left(\tau_{i}^{\alpha}-\frac{1}{2}\right)\left(\tau_{j}^{\alpha}-\frac{1}{2}\right) \\
& \left.+\frac{3}{2}\left(\tau_{i}^{\alpha} \tau_{j}^{\alpha}-\frac{1}{4}\right)\right] .
\end{aligned}
$$

In Eqs. (1) and (2), $\vec{S}_{i}$ is the spin at site $i$, while the orbital degrees of freedom are described by

$$
\tau_{i}^{a(b)}=\frac{1}{4}\left(-\sigma_{i}^{z} \pm \sqrt{3} \sigma_{i}^{x}\right), \quad \tau_{i}^{c}=\frac{1}{2} \sigma_{i}^{z},
$$

and $\alpha$ selects the cubic axis $(a, b$, or $c)$ that corresponds to the orientation of the bond $i-j$. The $\sigma$ 's are Pauli matrices acting on the orbital pseudospins $|x\rangle=$ $\left(\begin{array}{l}1 \\ 0\end{array}\right),|z\rangle=\left(\begin{array}{l}0 \\ 1\end{array}\right)$. Hence, we find a Heisenberg Hamiltonian for the spins, coupled into an orbital problem which is clock-model-like (there are three directional orbitals: $3 x^{2}-r^{2}, 3 y^{2}-r^{2}$, and $3 z^{2}-r^{2}$, but they are not independent) [14]. As we shall see, the Hund's rule coupling term (2) acts to lift the degeneracy. Next to this, we introduce another control parameter,

$$
H_{3}=-E_{z} \sum_{i} \tau_{i}^{c},
$$

a "magnetic field" for the orbital pseudospins, loosely associated with a uniaxial pressure along the $c$ axis.

The classical phase diagram of the spin-orbital model $H=H_{1}+H_{2}+H_{3}$, shown in Fig. 1, demonstrates the competition between the spin and orbital interactions similar to that found before in two dimensions [15]. It consists of five phases with staggered (two-sublattice) magnetic long-range order: (i) At large positive $E_{z}$, the orbital system is uniformly polarized along $x^{2}-y^{2}$. As no superexchange is possible in the $c$ direction, the $(a, b)$ planes decouple magnetically, and we recognize the two-dimensional (2D) antiferromagnet, called further AFxx, well known from the cuprate superconductors. (ii) At large negative $E_{z}$, the orbitals polarize along $3 z^{2}-r^{2}$, and the spin system is an anisotropic 3D antiferromagnet, called $\mathrm{AF} z z$. These two AF phases (AF $x x$ and $\mathrm{AF} z z$ ) are stabilized by the anisotropic superexchange which amounts to $4 J$ between $z$ orbitals along the $c$ axis, and to $9 \mathrm{~J} / 4(\mathrm{~J} / 4)$ between the $x(z)$ orbitals in the $(a, b)$ planes, respectively. In contrast, the Hund's rule $J_{H}$ stabilizes mixed-orbital (MO) phases in which both $\mathrm{AF}$ and ferromagnetic (FM) couplings occur. (iii) At large $J_{H} / U$ and $E_{z}<0$, a MOFFA phase is found, characterized at each site by orbitals, $|i \sigma\rangle=$

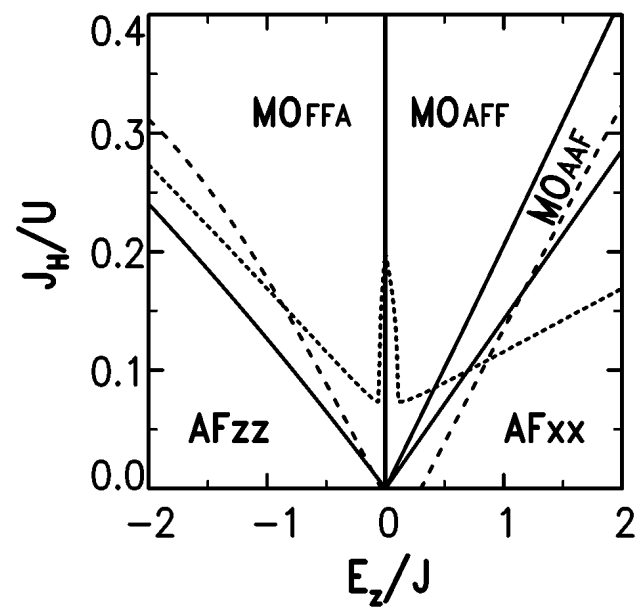

FIG. 1. Mean-field phase diagram of the spin-orbital model $(1)-(4)$ in the $\left(E_{z}, J_{H}\right)$ plane. Full lines indicate transitions between the classical states, while LRO is destroyed above the dashed (below the dotted) lines for the AF (MO) phases.

$\cos \theta_{i}|x \sigma\rangle+\sin \theta_{i}|z \sigma\rangle$, with the sign of $\theta_{i}$ alternating between the two sublattices in the $(a, b)$ planes. At small $\left|E_{z}\right|$ the orbitals stagger like $x^{2}-z^{2}, y^{2}-z^{2}$, $x^{2}-z^{2}, \ldots$, and point towards each other along the $c$ axis, which results in strong $(\sim J)$ AF interactions. In contrast, weak FM interactions $\left(\sim J_{H}\right)$ occur within the $(a, b)$ planes. (iv) A similar MO state, called MOAFF, is found at large $J_{H} / U$ and $E_{z}>0$, with the directional orbitals staggered in FM $(b, c)$ planes and $\mathrm{AF}$ order along the $a$ direction [16]. The MOFFA and MOAFF phases are degenerate at the line $E_{z}=0$, where $\cos 2 \theta_{i}=$ $-\left(1-\frac{\eta}{2}\right) /(2+3 \eta)$, and one is close to the KK phase (for which $\cos 2 \theta_{i}=-1 / 2$ [2]) in $\mathrm{KCuF}_{3}$, where, within the limitations of chemistry, the magnetic order appears to be equivalent to an ideal one-dimensional (1D) $S=1 / 2$ spin system. (v) Finally, the MOAAF phase with a small $|z\rangle$ amplitude, $\mathrm{AF}$ order in the $(a, b)$ planes and FM order along the $c$ axis, is stable between the MOAFF and AF $x x$ phases. Thus, we find the same ingredient as in the frustrated Heisenberg antiferromagnet (HAF): critical lines where different classical spin structures become degenerate. The present case appears to be more extreme, as even the effective dimensionality of the spin system changes because of the coupling to the orbital sector. The frustration manifests itself at the classical degeneracy point $M \equiv\left(E_{z}, \eta\right)=(0,0)$, where the orbitals may be rotated freely when the spins are AF, and the spins may be rotated freely within the FM planes of the MO phases. The same energy of $-3 J$ per site is obtained either in a 3D antiferromagnet with completely frustrated orbitals [consider $\left\langle\vec{S}_{i} \vec{S}_{i+\delta}\right\rangle=-1 / 4$ in Eq. (1)] or in a disordered spin system due to the orbital sector.

It is instructive to consider the stability of the classical phase diagram to Gaussian quantum fluctuations [15]. The collective modes can be calculated using, e.g., a random phase approximation (RPA) within the Green function 
technique [17]. Next to the Goldstone modes of the spin system, one finds optical modes corresponding to orbital excitations which occur both in the presence ("transverse") and in the absence ("longitudinal") of a simultaneous spin flip. The new feature is that the spin and transverse orbital excitations are coupled, so that fluctuations in the orbital sector also affect the spin sector. The approach of the critical lines is signaled by the softening of both longitudinal and transverse orbital modes. The (mixed) transverse modes give the dominating contribution to the renormalization of energy and magnetic order parameter. In the AF $x x(\mathrm{AF} z z)$ phase the lowest transverse mode softens along $\vec{k}=\left(\pi, 0, k_{z}\right)\left[\vec{k}=\left(k_{x}, 0,0\right)\right]$, and equivalent lines in the Brillouin zone (BZ), regardless of how one approaches the critical lines. Thus, these modes become dispersionless along particular (soft-mode) lines in the BZ, where we find finite masses in the perpendicular directions,

$$
\begin{aligned}
& \omega_{\mathrm{AF} x x}(\vec{k}) \rightarrow \Delta_{x}+B_{x}\left(k_{x}^{4}+14 k_{x}^{2} k_{y}^{2}+k_{y}^{4}\right)^{1 / 2}, \\
& \omega_{\mathrm{AF} z z}(\vec{k}) \rightarrow \Delta_{z}+B_{z}\left(k_{y}^{2}+4 k_{z}^{2}\right),
\end{aligned}
$$

with $\Delta_{i}=0$ and $B_{i} \neq 0$ at the $M$ point, and the quantum fluctuations diverge logarithmically, $\langle\delta S\rangle \sim \int d^{3} k / \omega(\vec{k}) \sim \int d^{2} k /\left(\Delta_{i}+B_{i} k^{2}\right) \sim \ln \Delta_{i}, \quad$ if $\Delta_{i} \rightarrow 0$ at the transition. An analytic expansion could not be performed in the MO phases, but the numerical results reported below suggest a qualitatively similar behavior.

We have verified that the above behavior of the soft mode results in large quantum corrections $\left\langle\delta S^{z}\right\rangle$ to the order parameter in all magnetic phases close to the critical lines. As an example, we show $\left\langle S^{z}\right\rangle$ in AF $x x$ and $\mathrm{AF} z z$ phases, being significantly lower than in a $2 \mathrm{D}$ HAF in a broad parameter regime (Fig. 2). Similarly, as in two dimensions [15], the LRO is overwhelmed by quantum fluctuations at particular lines, where $\left|\left\langle\delta S^{z}\right\rangle\right|=\left\langle S^{z}\right\rangle$ (Fig. 1). Unlike $\left\langle\delta S^{z}\right\rangle$, the RPA energies of the ordered phases show no divergence, with quite similar energy gains in $\mathrm{AF}$ and

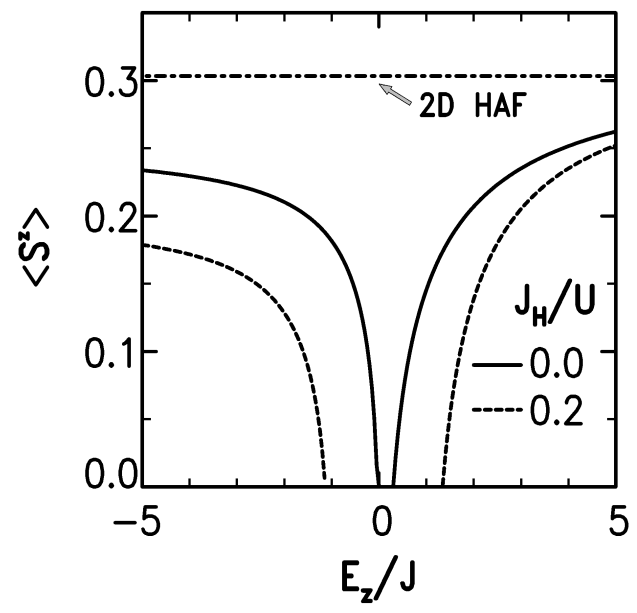

FIG. 2. Renormalization of AF LRO $\left\langle S_{i}^{z}\right\rangle$ in $\mathrm{AF} z z$ (left) and AF $x x$ (right) phases as functions of $E_{z} / J$.
MO phases of the order of $0.6 J$ [18]. We therefore believe that here RPA is as accurate as in the pure-spin HAF, and conclude that the classical order is destroyed by quantum fluctuations in the small $\left|E_{z}\right|$ and small $\eta$ region between dashed and dotted lines in Fig. 1.

Although the above theory is known to perform quite well in the simplest systems [19], it might be misleading in more complicated situations. For instance, the "finite mass mode softening" occurs also in the $J_{1}-J_{2}-J_{3}$ model where it is shown to be inconsequential in the large $S$ limit because of an "order out of disorder" phenomenon [6]. In contrast, low order spin-wave theory is blind for the quantum transition occurring in the bilayer model [4]. In all these cases, including ours, the quantum melting is promoted by the drastic enhancement of local fluctuations. It is then instructive to consider valence bond (VB) states [20] with the individual spins paired into singlets and the orbitals optimized variationally. As the energy of a singlet is lowest when the orbitals point along the bond, the optimal states with all singlets lined up in parallel (see Fig. 3) are (i) for $E_{z}>0$, singlets along the $a$ axis with orbitals close to $3 x^{2}-r^{2}$ ( $\mathrm{VB} a$, degenerate with the analogous $\mathrm{VB} b$ phase), and (ii) for $E_{z}<0$, singlets along the $c$ axis with orbitals $\sim|z\rangle(\mathrm{VB} c)$. Both optimize spin and orbital energy on every second bond, and have lower energy than the classical states close to the classical degeneracy.

Further, we included the leading quantum fluctuations in the VB states. A resonating $\mathrm{VB} c(\mathrm{RVB} c)$ state was obtained by making the singlets resonate along the $c$ axis, and its energy was calculated using the Bethe ansatz result for the 1D HAF, still including the orbital energies due to the bonds within the $(a, b)$ planes. The excitation spectrum is gapless, in agreement with Haldane's conjecture [21]. We also attempted to improve the $\mathrm{VB} a$ state by constructing the PRVB states, $\left|\Psi_{\square}\right\rangle \sim\left(\left|\Psi_{a}\right\rangle+e^{i \phi}\left|\Psi_{b}\right\rangle\right)$, from the singlet pairs along $a$ and $b,\left|\Psi_{a}\right\rangle$ and $\left|\Psi_{b}\right\rangle$. Surprisingly, more energy is gained instead in VB states in which plaquettes with singlet pairs $\left|\Psi_{a}\right\rangle$ and $\left|\Psi_{b}\right\rangle$, respectively, alternate and form a superlattice. The exceptional stability of these

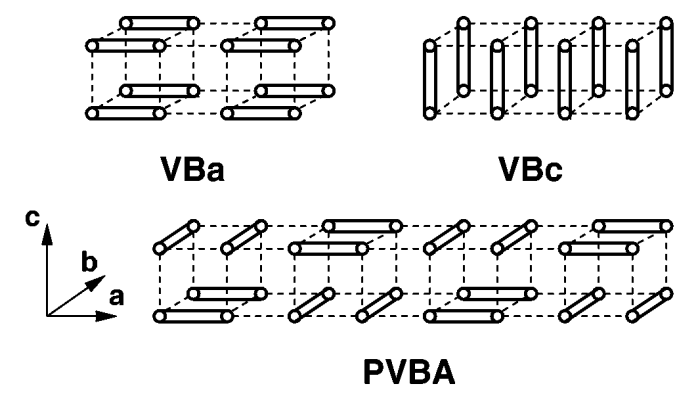

FIG. 3. Schematic representation of spin singlets (double lines) in the disordered states: $\mathrm{VB} a, \mathrm{VB} c$, and PVBA. In the PVBA state the plaquettes occupied by singlets $\| a$ and $\| b$ alternate in both the $(a, b)$ planes and the $c$ direction. 
(nonresonating) VB states is due to a unique mechanism involving the orbital sector. Unlike in the HAF, the bonds not occupied by the singlets contribute orbital energy, and this is optimized when singlets in orthogonal directions are connected. If $\eta<0.30$, this plaquette VB alternating (PVBA) state (Fig. 3) is stable at $E_{z}>0$, while another VB state, with single $(a, b)$ planes of PVBA phase interlayered with double layers of $\mathrm{VB} c$ phase (PVBI $c$ ), is stable at $E_{z}<0$. Finally, a state analogous to PVBI $c$, but interlayered along $a$ (PVBI $a$ ), occurs in between the PVBA and PVBI $c$ states. Thus, a spin liquid is stabilized by the orbital degeneracy over the MO phases with RPA fluctuations in a broad regime (Fig. 4). This resembles the situation in a 2D 1/5-depleted lattice [7], but the present instability is much stronger and happens in three dimensions.

In summary, we find strong theoretical arguments supporting the conjecture that quantum melting might occur in orbital degenerate Mott-Hubbard insulators. Why does it not occur always (e.g., in $\mathrm{KCuF}_{3}$ )? Next to the Hund's rule coupling $J_{H}$, the electron-phonon coupling $\lambda$ is dangerous. The lattice will react to the orbital fluctuations, dressing them up in analogy with polaron physics, and thereby reducing the coupling constant. In order to quantum melt $\mathrm{KCuF}_{3}$-like states, one should therefore look for ways to reduce both the effective $J_{H}$ and $\lambda$. We believe that this situation is encountered in $\mathrm{LiNiO}_{2}$ : Although the spin-spin interactions in the (111) planes should be very weakly FM according to the Goodenough-Kanamori rules, magnetic LRO is absent [22] and the system might represent the spin-orbital liquid. More strikingly, $\mathrm{LiNiO}_{2}$ is cubic at the $\mathrm{Ni}$ site and should undergo a collective JT transition, whose absence is actually an old chemistry mystery. Upon electron-hole transformation, $d^{7}$ low-spin $\mathrm{Ni}^{3+}$ maps on $d^{9} \mathrm{Cu}^{2+}$ in $\mathrm{KCuF}_{3}$, but with a difference in chemistry. While the $e_{g}$ hole in $\mathrm{KCuF}_{3}$ is almost entirely localized on the $\mathrm{Cu}$, the $e_{g}$ electron in $\mathrm{LiNiO}_{2}$ is

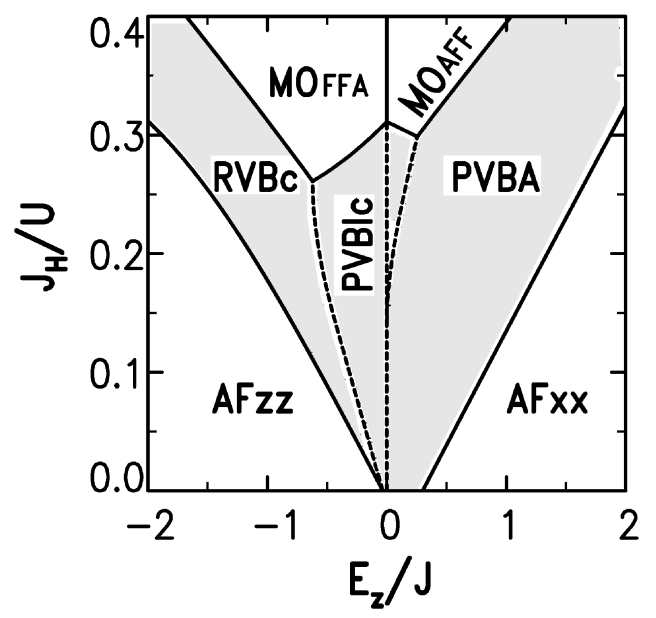

FIG. 4. The same as in Fig. 1, but including quantum fluctuations. The spin liquid ( $\mathrm{RVB} c, \mathrm{PVBI} c, \mathrm{PVBI} a$, and $\mathrm{PVBA}$ ) takes over in the shaded region between $\mathrm{AF}$ and $\mathrm{MO}$ phases. rather strongly delocalized over the $\mathrm{Ni}$ and surrounding $\mathrm{O}$ ions, which reduces both $J_{H}$ and $\lambda$, and explains the absence of classical ordering. A more precise experimental characterization of $\mathrm{LiNiO}_{2}$ is needed.

We thank D. I. Khomskii, M. Takano, and P. Horsch for stimulating discussions, and acknowledge the support by the Committee of Scientific Research (KBN) of Poland, Project No. 2 P03B 14408 (AMO), by the Dutch Academy of Sciences (KNAW) (JZ), and by ISI Foundation and EU PECO Network ERBCIPDCT940027.

*Permanent address: Institute of Physics, Jagellonian University, Reymonta 4, PL-30059 Kraków, Poland.

[1] E. Dagotto and T. M. Rice, Science 271, 618 (1996).

[2] K. I. Kugel and D. I. Khomskii, Sov. Phys. Usp. 25, 231 (1982).

[3] A. J. Millis and H. Monien, Phys. Rev. B 50, 16606 (1994); A. W. Sandvik and D. J. Scalapino, Phys. Rev. Lett. 72, 2777 (1994).

[4] A. V. Chubukov and D. K. Morr, Phys. Rev. B 52, 3521 (1995).

[5] P. Chandra and B. Doucot, Phys. Rev. B 38, 9335 (1988); A. Moreo et al., Phys. Rev. B 42, 6283 (1990); N. Read and S. Sachdev, Phys. Rev. Lett. 66, 1773 (1991).

[6] A. Chubukov, Phys. Rev. B 44, 392 (1991).

[7] K. Ueda et al., Phys. Rev. Lett. 76, 1932 (1996); M. Troyer et al., Phys. Rev. Lett. 76, 3822 (1996); S. R. White, Phys. Rev. Lett. 77, 3633 (1996).

[8] A. I. Liechtenstein et al., Phys. Rev. B 52, R5467 (1995).

[9] P. Fulde and I. Peschel, Adv. Phys. 21, 1 (1972); Y. Y. Hsieh and M. Blume, Phys. Rev. B 6, 2684 (1972).

[10] C. Castellani et al., Phys. Rev. B 18, 4945 (1978); 18, 4967 (1978).

[11] T. Mizokawa and A. Fujimori, Phys. Rev. B 54, 5368 (1996).

[12] D. L. Cox, Phys. Rev. Lett. 59, 1240 (1987).

[13] W. Weber, Z. Phys. B 70, 323 (1988).

[14] Physically this could correspond with the perovskite planes as in the high $T_{c}$ cuprates, squeezing the $\mathrm{Cu}$-apical $O$ bond length. Of course, $E_{z} \neq 0$ in the actual high $T_{c}$ materials; see, D. I. Khomskii, Physica (Amsterdam) 171B, 44 (1991).

[15] J. Zaanen, A. M. Oleś, and L.F. Feiner, in Dynamics of Magnetic Fluctuations in High Temperature Superconductors, edited by G. Reiter et al. (Plenum Press, New York, 1991), p. 241; L.F. Feiner, A. M. Oleś, and J. Zaanen, J. Magn. Magn. Mater. 140-144, 1941 (1995).

[16] The MOAFF phase is degenerate with a MOFAF phase which has the orbitals staggering within the $(a, c)$ planes.

[17] S. B. Haley and P. Erdös, Phys. Rev. B 5, 1106 (1972).

[18] The energy in the MO phases for $E_{z}>0$ was estimated using the averaged crystal field over the two sublattices.

[19] S. Chakravarty, B. I. Halperin, and D. R. Nelson, Phys. Rev. Lett. 60, 1057 (1988); Phys. Rev. B 39, 2344 (1989).

[20] A. Auerbach, Interacting Electrons and Quantum Magnetism (Springer, New York, 1994).

[21] F. D. M. Haldane, Phys. Lett. 93A, 464 (1983).

[22] K. Hirakawa et al., J. Phys. Soc. Jpn. 54, 3526 (1985); K. Yamaura et al. (unpublished). 